\title{
Nachträge und Berichtigungen.
}

Soeben nach dem Abschlusse des ganzen Druckes schickte mir Seuffert sein Exemplar von 0 , weil ihn erst meine Angaben auf dem achten Bogen die Sonderstellung erkennen liessen; es stimmmt im Äusseren völlig zu $O^{1}$, ist in der Zeilenbrechung mit $O^{1}$ ganz identisch'), während sich in $O^{2}$ mitunter kleine Verschiedenheiten finden. Trotzdem ist es nicht $O^{l}$ und nicht $O^{2}$, sondern steht mit seinen Lesarten zwischen beiden und beweist, dass die Weygandische Buchhandlung den Julius wenigstens dreimal mit der Jahreszahl 1776 abdrucken liess, wenn nicht das Exemplar, welches Sauer seiner Ausgabe zu Grunde legte; nach den gelegentlich bemerkten Abweichungen von $O^{2}$ einen weiteren vierten Abdruck repräsentiert. Seufferts Exemplar, das ich mit $S$ bezeichnen will, da $O^{3}$ täuschen könnte, ist wirklich ein nener Abdruck; folgende Lesarten zeigt es gegen $O^{1}$ und $O^{2}$ :

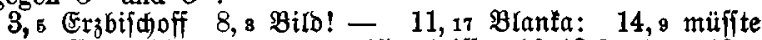
14,18 grofien 14,16 hören: - 15,1 heifft 16,12 ftünde, 19,9 fodert 21,9 er. $-25,1$ hat: $-34,11$ Julius. - 37, 9. 10 Blüf 39,2 hat. - 39,7 5irten, 42,14 Bilonis 44, 28 geben! 48,11 Miäbdens! - bieje 49,12 werben, 52, 2 Entfílus 70,8 Eiferjuตt 75,12 überwunden; - 81,2 mübe! $-81,4$ uner: mefflidfeit 82,3 geftehn: - 87,5 Regende: - 104, s jenn? 108,11 glDuben. [statt glauben.] 108,14 läift 113,12 gemäffígt) 114,11 an! $O^{1}$ an!) $S$ an.) $O^{2} \quad 115$, s Beidjäp ! - 124, 7 Wejen; -

Es sind freilich nur unbedeutende Verschiedenheiten, sie müssen jedoch der Vollständigkeit halber notiert werden. Ich teile nun jene Stellen mit, an denen $S$ im Richtigen oder Falschen gegen $O^{1}$ mit $O^{2}$ stimmt; überall, wo nichts bemerkt ist, stellt sich $S$ zu $O^{1}$, falls zwischen $O^{1}$ und $O^{2}$ ein Unterschied besteht. $\mathrm{Zu}$ gleich seien ein paar Versehen berichtigt.

1) Nur an einer einzigen Stelle ein ganz kleiner Unterschied.

Litteraturderkmale des 18 u. 19. Jahrh. 32. 
3, Эưtinenflofters. [der Punkt steht auch $O^{2}$ ] 5, a Palaft.

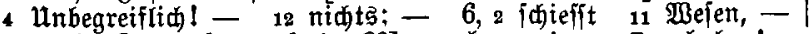
gebrüft, [so steht auch in $O^{2}$ ] 16 fennen! - 7,7 haben! [14 That, $O^{1}$ ist zu streichen] 17 Mann!" 8, 1 Sieffen / gieffen 8 Standes! - 11 Strid, - 14 Blanfa. - | ftille! - 15 gemis 17 Brinz! - 9,1 nid); - 2 Bilonis, | bacte, 6 Bilonis, 7 bem 14 tragen; - [so auch $O^{2}$ ] 16 biesmal 10,1 Fürft; 2 Itnglüf! - 3 f́tillen! - $\mid$ mir! - 5 Glüf 10 laff' 19 eing; 14 warten. - 15 mid, - 11,4 meig 6 Entmurfs! - 10 muffte, 14 fenn. - I Jhrem 20 Entidlus 12, s Ganze! - 4 Bhilos fophie! - 8 Entid)lus ein Entfdlus 15 läfjt 13,8 Mann! 6 i $;$; - 8 Sörper 12 Drat 14 (Eharafter. - 14,5 meifft Blanfa; - 8 fite $\mid$ fdiffte. 9 Müffunft 15 genannt; - $[16$ hören; - $\left.O^{2}\right]$ 15, 2 setze hinzu: Rahmen $O^{1} S_{7}$ Tapferfeit; -14

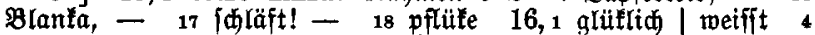
Sфooffe 6 aufriøten - \&aff' [zu einer Hälfte gleich $O^{1}$, zur

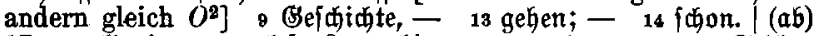
17,1 wollen! - \& gelejen? 18 bitt, - 14 gelogen; - 18 Shön= heit! [so auch $O^{2}$ ] 18, s Bejez 4 Beidling. - 8 eingefühtt; [o setze man zu: er] es $O^{2}$ ] 11 lezte 12 fumpfinten 13 zulezt 18-14 Jhnen, - 14 [setze man zu: fie $O^{1}$ ] [ blos 15 verbrieffit 20 BefGimpfung. - 19,2 fenn; 4 ßalaft 5 ftoffe 14 Eș

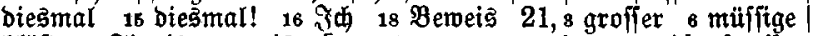
bläpt 7 Firnis 9 mets [17 setze man zu: is vom Abschreiber unterstrichen] 22, 3 Fuffe) 6 beiffen. 23, $7 \mathfrak{D} \mathfrak{u} 9$ meis, $\mid$ weis, 14 Bifdoff 24, 7 Stetigfeit? - 8 will; -9 Entidlteffungen, 15 reiffen, 25, 3 Seroinnl- / Entidilus 26, 8 [Stretde $O^{1}$ zu tilgen] 14 [man setze zu: ifm $O^{1} S$ ] | verbriefft, 27,4 wuffte, 6 gröfftes $\left[=O^{2}\right]$ Bergnügen $\left[=O^{1}\right] \quad 29,7$ Berfd lieffen 12 ist zu lesen: präde. $O \mid \mathfrak{D a} 5, O^{2} S$ ] $14 \mathrm{im}$ Texte lese man [25] 30,1 bloz a verbessere man: erriethe. $O^{1} S$ perriethe. $O^{2} \quad z^{\mathfrak{u}}=$ jammentiöfft, 31, 1 Quentin 7 blos 17 Metfit 19 jehen? Ment nur 32, 9 gemis, 33, 2 (fitfft $\left[41 . D_{\mathrm{ir}}, 0\right] 34,3$ Siffies $\left[=O^{2}\right]$ aber $\mathfrak{B}_{\text {ittrea }}\left[=O^{1}\right]$ | ergiefien 9 lese man: zu[ammen genom= men $O^{1}$ zufammen genommen, $O^{2} S \quad 11$ Eaecilia? - 35, 2 Bers gnügen. - 7 zuffieffen 14 unferer 15 Parabiefe. - 36, 1 blog 4 weis s Battin! - 16 wets 37,4 lese man: verlaffen, $O$ । alles, $O^{2} \quad 8$ Ertlärung. - 19 lese man: ftünbe, $O^{2} S \quad 38,7$ meis

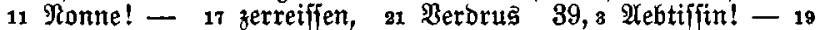
(Beridiefft 40, s bies 6 heifft 11 lese man: jehen? - $O^{2} S$ । jeben? $-O^{2} S{ }_{12} \mathfrak{R u h e}$ - 14 gröfiten 17 Seinen 41,1 ergänze man: zweite $O^{1}$ [jwente $O^{2} S$ ] s hat! - 14 Afles; -

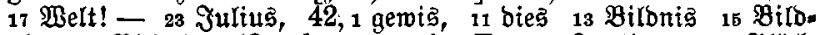
nis 21 Bilonis 43, 7 lese man im Texte: $\Im \mathfrak{u}$ li feligfeit 15 fönnten $17 \mathfrak{B i l} b .-20$ lese man im Texte: $\mathfrak{B}$ lanca. und in den Anm. $B \mathfrak{a} \pi \mathfrak{f} a, O^{2} S$ geträumt? - 44,4 eins!- 
7 minielten! - 20 mid ! - 45,1 unierm 5 grojien 9 fülift 12 mid! - 14 wahrhaftig! - 16 wär' 46, 10 Đoffrung, 47, lese man: \$ferbe, $O^{2} S$ s Erbe! - 4 lese man: flein; - $O^{2} S \mid$

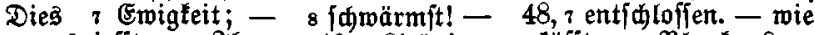
18 ausgiefft, 18 Эhrem 49,2 \&iebe! 18 getüfft. 19 Blanfan?-

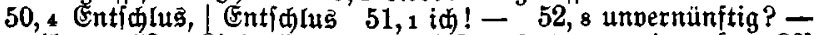
18 ihren 53, s \&iebestiebgen $\left[=O^{1}\right]$ einfdlummert! $-\left[=O^{2}\right]$

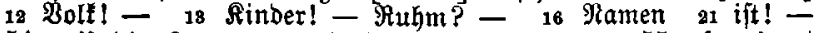
54,16 Itnding? - 17 pereint! - 19 Seufzer; - 55, 1 fenn!-1 muffite o vergifte! - 7 lese man: Sie, $O^{2} S$ s entidieden! 18 \&uft, 15 Sdluffe 16 Bormurf? - - [der Gedankenstrich in $O^{1}$ ist ungewöhnlich lang] $18 \Re$ egenbogen! - | ऽ中 | Riebe; 56,1 Trägheit! - 7 Entfablieffungen - 11 Morgen! - 28 hin! - 57, 8 hin! - 6 wendet; - 8 Gerz! - 8 bod: 12 Monat? - 18 Gut, - | Monat! - 58,9 Traurigfeit! bies 11 aufhören, - 59, a Eaecilta? - 8 befohlen; - 21 ver = banfen, - 60, 7 Gotte. - 16 Geheimnis 61,9 foll; -12 füh $=$ ren: - 62, 3 fönnen. - 9 muffite 11 empfinden. - 13 jeyn? 63,2 unferm a nennen? - 64,6 bebeftem 9 entblöfitem 15 furzem 65, 22 laffen! - 66,1 Sterbenden. - 16 א̃inder, 19 Saus?" - 67, 16 Segen 68,2 lafft 4 allem, 6 wüfften, 7 zerreifft 9 zerreiffien 10 fanft. - 11 Măjfítgung 12 ergänze man : bas $O^{1}$ [bas $\left.O^{2} S\right] 19$ faltem $/$ verwundet; $-69,8$ foden= bem 11 Eurem 15 groffe 17 wilft: $-70,1$ Safft $T$ eint Nidts! - 8 eiferfüdtig? - - Riebe: $-\mid$ heifft 71,11 muffit 12 Dein 19 hetvorragen; - 20 vergeffne 72,5 Myrtengebüj申; 8 gefunfen; - 21 bies 22 Mäbdens, - 1 Baters̄, - 73, ftehft? - 6 aufführt, - 6 Shauplaz! (fie 9 lafft I bies 74,8 auffer 10 łommen! - 13 reden! - 17 weifft 75,8 jenn! - 76,1 5arnifक? - 2 groffe 6 gründlid; - 8 Mörber! 11 heifft 19 wolte? - $\mid$ Menn | will! - 77, \& Ehre: - $\mathbf{3}$ id 7 Thoren? - | rajejt! - 9 Shmadheit, - 15 idrazen! 78 , 10 fan! - man lese: jolft $O$ 18 ift! - J゙ 79 , a nehme! 4 Gintänbeln! - 5 verfolgen, $-80,9$ verlieren! - 10 brin=

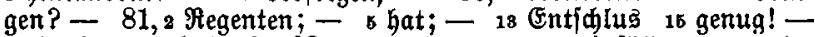
16 barin, 18 haben? 82 , entfübren; - 9 einfält; -10 be $=$ rührt! - 11 lief́: - 83,11 verlaffen! 12 Freunbe! - 85, 8 Friebe? - 5 jtraft? 6 Tob! Lieblingsgebanfe? - 14 lieft 18 eine 19 Füffen; - 86, 4 $\mathfrak{A}$ ebtiffin. 87, s meinen? - 10 mäfiłge 18 Emigfeit! - 88, s gegrüfft, 16 Thränen! - 20 er= finnenl - 89,5 Todter, | wilb! 18 meinem 15 heifft 90,10 Betrübnis 91,11 zerreiff' 12 willen! - 92,16 Beit!- 93, 5 burfirren; - 12 lese man: $O^{1} S 19$ ergänze man: dię́ $O^{1}$ [bieș $O^{2} S$ ] 94, 1 Bäter; - 8 fah! - 9 Agneje! - 95, 1 braudt: 7 Erbbegräbris 10 fino. - 11 haben; - 16 Slü山-

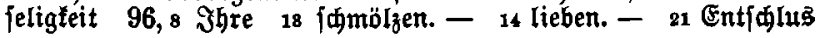


97, 1 reife! - | Entílus \& beitehn;- 10 Bater. - 14 f fieue; - 21 Mine, 28 Deinen 98,10 baş 12 Segen | pergröffert 19 Iöjđ̈en? 21 Mine! 99,2 Segen \& Segen 100,6 über! ? Meifft 16 bem 16 fagen: 17 Tarent! - 101, 20 bag | wufft' 102, 9 Bater; - நimmel! - 13 Abgott. - 15 jener, und 16 bie fera 103, fühlte! - 10 bas / fajift. - 13 Straffe 104, 4 erheben! 16 warten. - 17 tönnen. Wie 105,18 gebetet; 19 Gartenthür! Eine | Thomas! - 20 veríblieffen, 106,1 ge= zogenen o (reifft 7 Bruber! - | Gindern. - 8 vor! 9 Iarent? 18 Blanfa, Blanta! 19 er, - 20 Gebeine! - 107, 7 Greis; - 8 maden! 10 Dummfopfs! - 108, 9 2II wiffens, 18 tragen! - 20 Sie! - 111, 1 mein \& Entwürfe! - 7

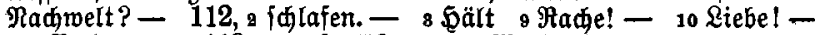
12 Bube. - 118, s aufgelöftem 7 Mörber! 9 erwaden; 11 pernidtet! - 16 neuem 17 Mörderin! (Paufe) 24 fan. $25 \mathrm{ab}\left[=O^{1}\right]$ (füffit $\left[=O^{2}\right] \quad 26$ Freuben $\left[=O^{1}\right]$ (Eüfift $\left[=O^{2}\right]$ 28 laff $^{\prime} 114,8$ tröften; - 115,6 Stjooffe 18 Derbinbung! 28 nidit! - 116, s herabjtoffen - 4 Reidnam! 11 \&iebe? 12 நülfe! - 1s Reoparb, - Tiger, - Bär! 117, 11 liefft 12 शुerrü̈t? - 18 lieffit 17 mütendem 18 jehe! 118, 6 ent= fliebt! - 18 Sqüfer! 16 erröthe! 119,10 Priefter. 16 Tarent! 120, 19 Pantomime, 20 zuftieffe) 121, 15 Sünbe! - 17 fah; 19 Freuben! 20 Jhr 122,1 gelejen: - 13 habe! - 124,4 Galte. - † id ? - 0 fomme! 12 Sünde: - 16 Bergnügen! - 125, 1 bies 6 anbern! - 11 Gutbo? - 126, 7 Bruber!

In $S$ wurde nach dem Gesagten hauptsächlich die Interpunktion und Orthographie von $O^{\mathrm{i}}$ geändert, eigentliche Fehler haben sich nicht eingeschlichen; $O^{2}$ schreitet auf dem von $S$ eingeschlagenen Wege weiter fort, enthält aber eine Reihe von Irrthümern, welche den Text entstellen. Den S. XXXIII gegebenen Stammbaum müssen wir nun so richtig stellen:

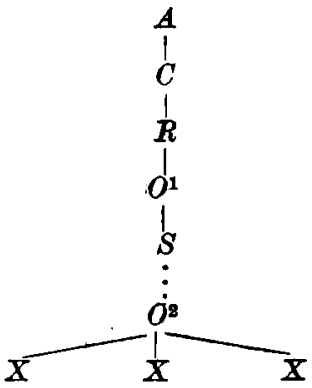

Es ist möglich, dass sich zwischen $S$ und $O^{2}$ noch Mittelglieder werden aufweisen lassen, worauf die Lesarten in Sauers 
Ausgabe hindeuten ${ }^{1}$ ). Vielleicht fühlen sich die Besitzer von Originalausgaben des Julius durch diese Darlegung veranlasst, ihren Text zu prüfen.

$O^{1}$ und $S$ gleichen sich im Äusseren fast völlig; sie haben dieselbe Columnenverzierung, einen Lorbeerstab [?], während $O^{2}$ nur einen einfachen Strich zeigt, denselben Schlussstrich auch am Ende des Stückes. Dagegen hat $O^{1}$ nach dem ersten Aufzuge $\mathrm{S}$. 30 einen ungleich dicken Doppelstrich, $S$ wie $O^{2}$ einen einfachen; dieser einfache Strich erscheint nach dem zweiten Aufzug in $O^{1}$ und $S$, während $O^{2}$ einen ganz kurzen Strich aufweist (S. 54); zwischen den übrigen Aufzügen fehlt ein solcher Strich in allen drei Ausgaben. [3. März 1889.]

1) Ich greife nur eine Stelle heraus; 6,3 lesen wir in $A B O^{1} S$ (abgesehen

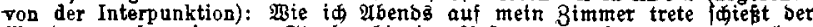
Shonb nur eben ein paar Stralen binein, $O^{2}$ dagegen und Sauer: $n$ in ebent. Andererseits lesen $118,13 A O^{1} S$ und Sauer wennebr , wâhrend $O^{2}$ toenn efr hat; dies scheint darauf hinzudenten, dass jenes von Sauer seinem Abdruck zu Grunde gelegte Erémplar von $O$ weder mit $S$ noch mit $O^{2}$ identisch sei, uns also eine Weiterentwickelung des Textes von $A$ \& $O^{2}$ reprasentiere.

Le mberg am 27. Juni 1888.

Richard Maria Werner. 